\title{
Remoción de Fósforo en un Sistema de Humedales Artificiales a Escala de Laboratorio
}

\author{
MARÍA IVONNE REYES LUZ, ALEJANDRO GUIDO ZÁRATE, \\ SONIA GABRIELA CARRILLO NÚÑEZ, CARMEN DURÁN DE BAZÚA ** \\ a Departamento de Ingeniería Química, Facultad de Química, UNAM, Paseo de la Investigación Científica s/n, \\ 04510 México D. F., México. Tel. 5622-5300 al 04, Fax: 5622-5303 \\ *Correspondencia: mcduran@unam.mx
}

Recibido: 15 marzo 2011 / Aceptado: 14 septiembre 2011

\section{Resumen}

Se estudió la remoción de fósforo en cuatro reactores biológicos empacados con escoria volcánica (tezontle) que simulan la parte radicular de un humedal artificial. El diseño experimental incluyó el efecto de la planta y sus réplicas (reactores $R A_{1}, R B_{1}$ con planta y $R A_{2}, R B_{2}$ sin planta), así como la concentración inicial de contaminantes medida como demanda química de oxígeno (relación C:N:P de 30:1:0.04 equivalente a $450 \mathrm{mg} D Q O / L$ y 15:1:0.04 equivalente a $225 \mathrm{mg} D Q O / L)$ en la asimilación de fósforo. El agua residual sintética se elaboró con sacarosa como fuente de carbono, $\mathrm{C}_{12} \mathrm{H}_{22} \mathrm{O}_{11}$; sulfato de amonio como fuente de nitrógeno, $\left(\mathrm{NH}_{4}\right)_{2} \mathrm{SO}_{4}$ y fosfato de sodio dodecahidratado como fuente de fósforo, $\mathrm{Na}_{3} \mathrm{PO}_{4} .12 \mathrm{H}_{2} \mathrm{O}$. Para simular periodos de $16 \mathrm{~h}$ de luz y $8 \mathrm{~h}$ de oscuridad se emplearon 8 lámparas de tubos fluorescentes de $30 \mathrm{~W}$. Se colocaron tres electrodos comerciales (Ag/AgCl-Pt) para medir potenciales de óxido-reducción, pOR, a diferentes profundidades $(2,10$, $30 \mathrm{~cm}$ de la superficie de cada reactor). Se plantaron brotes de Phragmites australis (carrizo) en los reactores $R A_{1}$ y $R B_{1}$. Al alcanzar la estabilidad se midieron los parámetros de fósforo total, $D Q O$, potenciales redox, $\mathrm{pH}$ y temperatura. Los resultados muestran que en todos los reactores hubo remoción de fósforo y que fue significativamente mayor con planta ( $R A_{1}$ y $R B_{1}, 93 \%$ en promedio para ambos reactores) ( $\left.p<0.05,0.01\right)$. No hubo una diferencia significativa $(p<0.05,0.01)$ entre la disminución de la $D Q O$ y la de $P$ para las dos relaciones $C: N: P$. La remoción de la DQO para el diseño experimental del efecto de la presencia de la planta y de la concentración inicial de DQO sí fue estadísticamente diferente debido a la presencia de la hidrofita, así como a la concentración inicial de carbono ( $p<0.05$ para la presencia de la planta y $p<0.05,0.01$ para la concentración inicial de carbono), siendo ligeramente mayor en aquellos reactores con planta, indicando que ésta sí tiene un efecto depurador adicional a las comunidades microbiológicas que proliferan en los reactores.

Palabras clave: fósforo, humedales artificiales, escala de laboratorio.

\section{Removal of Phosphorus in a Laboratory Scale System of Artificial Wetlands}

\section{Abstract}

Removal of phosphorus in four packed biological reactors simulating the root zone of an artificial wetlands was performed. Reactors packing was volcanic slag (tezontle in the Aztec language). The experimental design included the effect of the macrophytes (reactors $R A_{1}$ with plant, and $R A_{2}$ without plant), and its replicas $\left(R B_{1}\right.$ with plant, and $R B_{2}$ without plant), as well as the initial concentration of pollutants measured as chemical oxygen demand

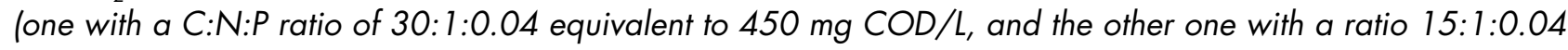
equivalent to $225 \mathrm{mg} C O D / L)$ in the phosphorus assimilation. Synthetic wastewater was made using sucrose as carbon source, $\mathrm{C}_{12} \mathrm{H}_{22} \mathrm{O}_{11}$; ammonium sulfate as nitrogen source, $\left(\mathrm{NH}_{4}\right)_{2} \mathrm{SO}_{4}$, and dodecahydrated sodium phosphate as phosphorus source, $\mathrm{Na}_{3} \mathrm{PO}_{4} .12 \mathrm{H}_{2} \mathrm{O}$. Light was obtained from 8 lamps of $30 \mathrm{~W}$ fluorescent tubes automatically controlled to simulate light periods of $16 \mathrm{~h}$ and $8 \mathrm{~h}$ darkness. Three commercial electrodes $(\mathrm{Ag} /$ $\mathrm{AgCl}-\mathrm{Pt})$ to measure oxidation-reduction potentials, $\mathrm{pOR}$, at different depths were placed to evaluate the redox 
potential differences $(2,10,30 \mathrm{~cm}$ from the upper reactor surface). Shoots of a Phragmites australis macrophyte were planted in the reactors $R A_{1}$ and $R B_{1}$. Once stability was reached total phosphorus, $C O D$, redox potentials, $\mathrm{pH}$, and temperature. Results show that all reactors had phosphorus removed, and the removal was significantly higher in the reactors with plant $\left(R A_{1}\right.$ and $R B_{1}, 93 \%$ in average for both reactors) ( $\left.p<0.05,0.01\right)$. No significant difference $(p<0.05,0.01)$ was found between the $C O D$ reduction and phosphorus for the two experiments with 450 and $225 \mathrm{mg} C O D / \mathrm{L}$. Removal of COD for the experimental design carried out (effect of the macrophyte and the initial COD concentration) in both cases was statistically different due to the presence of the plant, as well as the initial carbon concentration $(p<0.05$ for the plant presence, and $p<0.05,0.01$ for the initial carbon concentration), being slightly higher for those reactors with plant, indicating that they do have an additional effect to the microbiological communities proliferating in the reactors.

Key words: phosphorus, artificial wetlands, laboratory scale.

\section{Introducción}

En México, el problema de la contaminación del agua y su disponibilidad para las diversas actividades económicas y productivas del país es cada vez más creciente (1), lo cual ha obligado a la búsqueda de alternativas de solución económicamente viables (16). Se estima que aproximadamente el $80 \%$ de las aguas residuales generadas son descargadas al ambiente sin ningún tratamiento adecuado (3).

A nivel mundial, la preocupación por los problemas de contaminación del agua se dio con mayor ahínco a partir de la década de los setenta del siglo XX en Europa y países como Estados Unidos de América, Canadá, Gran Bretaña y Japón, en donde la urbanización y la industrialización creciente se vieron acompañadas de graves problemas de contaminación del agua. En las regiones menos desarrolladas, los desechos de las poblaciones constituyen una amenaza para la salud pública y ponen en peligro el uso ininterrumpido de reservas de agua (4).

En el agua residual, el fósforo puede provenir de varias fuentes, principalmente por el uso de los detergentes a los que todavía se les adicionan fosfatos, generando problemas en los cuerpos de agua ya que el fósforo en grandes cantidades provoca daños al alterar el equilibrio de los mismos.

Debido a que el fósforo es un nutriente esencial para los seres vivos, los microorganismos presentes en los procesos de tratamiento pueden asimilarlo e incorporarlo a sus tejidos celulares. Cuando el fósforo no es removido de las aguas residuales y cuando éste llega en concentraciones relativamente altas a los cuerpos de aguas, este nutriente permite el crecimiento y desarrollo de una gran cantidad de algas y otros microorganismos. Al morir, estos microorganismos tienden a flotar en la parte superficial del agua limitando el paso de la luz solar y, con ello, limitando que la fotosíntesis se lleve a cabo de manera normal, lo que da lugar a una disminución en la concentración de oxígeno dentro del cuerpo de agua, provocando la muerte de peces y otros organismos superiores. A este fenómeno se le conoce como "eutrofización".

Es necesario que el fósforo se elimine de las aguas residuales antes de que éstas se descarguen a los cuerpos de agua causando al fenómeno antes descrito. El empleo de tecnologías alternativas como los humedales artificiales puede ser la solución a este problema. Sin embargo, es necesario estudiar los mecanismos de remoción que se presentan dentro de estos sistemas para poder entender mejor su funcionamiento y aplicar criterios de diseño basados en la información generada.

Los humedales artificiales, $\mathrm{HA}$, son sistemas de tratamiento de aguas residuales que imitan la actividad depuradora realizada por los humedales naturales. A grandes rasgos, un HA se construye haciendo una excavación de forma rectangular que es impermeabilizada y después rellenada con uno o varios materiales de empaque como arena, grava o tezontle que funcionan como filtro para el agua y como soporte para las plantas que son sembradas en él. El material de empaque también funciona como soporte para la gran cantidad y diversidad de microorganismos (bacterias principal- 
mente) que se desarrollan sobre él y sobre las raíces de las plantas y que contribuyen a la depuración del agua degradando la materia orgánica. Desde el punto de vista de la ingeniería, un humedal artificial puede conceptualizarse como un reactor empacado donde ocurren reacciones químicas y biológicas, así como procesos físicos sobre la superficie del empaque.

Los HA imitan los procesos que ocurren en los humedales naturales, con las variantes de que en los HA se regulan ciertos parámetros como la cantidad de agua a tratar, el régimen de flujo y la calidad de agua obtenida. Los contaminantes en los HA se remueven a través de una combinación de procesos físicos, químicos y biológicos, incluyendo la sedimentación, precipitación, adsorción, asimilación por las plantas y transformaciones bioquímicas mediante los microorganismos presentes en forma de película sobre el material de soporte (5). Dentro de un HA, las áreas próximas a las raíces de las plantas son aerobias, especialmente durante el día cuando la actividad fotosintética de la planta favorece la generación de oxígeno, mientras que las más alejadas de ellas o durante la noche cuando las plantas respiran como cualquier organismo aerobio, consumen el oxígeno presente en sus alrededores y las condiciones de las áreas de todo el reactor son anaerobias, con excepción del área en contacto con el aire del ambiente, donde por difusión molecular este elemento se difunde al reactor, permitiéndose así que se desarrolle una gran variedad de microorganismos dentro del lecho, incluyendo no sólo bacterias, sino también protozoarios, rotíferos, nemátodos, etc., mismos que contribuyen a la remoción de los contaminantes (1).

Se ha encontrado que los humedales son efectivos para el tratamiento de la DBO, sólidos suspendidos, nitrógeno y fósforo, así como la disminución de la concentración de metales, compuestos químicos, orgánicos y patógenos ya que muchos de los grupos microbianos de los humedales son predadores de los organismos patógenos $(6 ; 7)$.

Tomando en cuenta que los humedales construidos o artificiales son ecotecnologías económicamente viables, de gran capacidad para la remoción de contaminantes y que no necesitan de grandes costos en su operación y mantenimiento, los humedales son una buena opción para implementarse en comunidades pequeñas o rurales para el tratamiento de las aguas residuales que éstas generan y que, después de ser tratadas, puedan utilizarse para riego en las zonas que no cuentan con el capital para suministrar agua potable para riego, además de que son estéticamente agradables a la vista.

Un humedal está compuesto por agua, "sustrato" o material de soporte, plantas vasculares, residuos de la planta, invertebrados (larvas y lombrices) y una gran variedad de microorganismos (en su mayoría bacterias), otro componente importante de un humedal artificial es la capa impermeable que evita que el agua contamine el subsuelo y las aguas subterráneas, quedando aislado de los alrededores. El humedal sólo cumple con la función de tratar el agua. La hidrología es el factor de diseño más importante en un humedal artificial porque es este factor el que determina en mayor medida muchos de los mecanismos por los cuales se remueven los contaminantes dentro de un HA. Se requiere un soporte debido a que las macrofitas no cuentan con un medio de soporte, el material de empaque utilizado es generalmente inerte como la arena, la escoria volcánica, piedras o materiales de las propias zonas que resulten económicas y viables para su utilización (8). El material de soporte es también muy importante para los microorganismos, los cuales juegan un papel muy importante en la degradación de la materia orgánica, ya que pueden existir transformaciones microbianas aerobias (es decir, requieren oxígeno libre o molecular) mientras otras transformaciones son llevadas a cabo en condiciones anaerobias (tienen lugar en ausencia de oxígeno libre o molecular). Los microorganismos presentes en el material de soporte de un humedal y en la zona de la rizosfera de las plantas son los responsables de llevar a cabo la degradación biológica de la materia orgánica. Estos consumen el $\mathrm{C}, \mathrm{N}$ y $\mathrm{P}$ disueltos en el agua y parte de estos nutrientes los incorporan a su tejido celular (1), para llevar a cabo los procesos metabólicos, como su crecimiento y reproducción. El mayor beneficio de las plantas es la transferencia de oxígeno a la zona de la raíz. Su presencia física en el sistema (los tallos, raíces y rizomas) permite la penetración al medio de soporte y transporta el oxígeno de manera más profunda 77 $9 ; 10)$, de lo que llegaría naturalmente a través de la difusión.

Esta investigación tiene como objetivo probar en un sistema de humedales artificiales a escala de labora- 
torio el efecto de las macrofitas en la eliminación del fósforo presente en aguas residuales.

A continuación se presenta la metodología seguida y los resultados obtenidos.

\section{Materiales y Métodos}

El sistema experimental a escala de laboratorio se montó en un laboratorio donde la temperatura se mantuvo relativamente constante $\left(22 \pm 2^{\circ} \mathrm{C}\right)$. El sistema constó de cuatro reactores de $30 \mathrm{~cm}$ de diámetro y $35 \mathrm{~cm}$ de altura empacados con escoria volcánica (tezontle) previamente lavada y esterilizada a $121^{\circ} \mathrm{C}$ por $30 \mathrm{~min}$ (1), sirviendo como soporte para las plantas y para los microorganismos. La escoria volcánica tiene un tamaño de partícula específico que permite un adecuado flujo del agua residual (2). Su granulometría estuvo distribuida como se muestra en la Tabla 1. El sistema experimental estuvo compuesto por los reactores $R_{1}$ (reactor con planta) y $R_{2}$ (reactor sin planta) y sus réplicas ( $R B_{1}$ con planta y $R B_{2}$ sin planta), que funcionaron como humedales artificiales de flujo vertical descendente (HAFV). Las Figuras 1, 1a, $1 b$ y 2 muestran a detalle el sistema experimental empleado. Estos reactores se alimentaron con agua residual sintética $(11 ; 12)$, con un flujo de alimentación continua de 7 litros por día manteniendo condiciones de inundación (un cm debajo de la superficie). Se implementó un sistema para la iluminación de los reactores manteniéndose con $16 \mathrm{~h}$ de luz y $8 \mathrm{~h}$ de oscuridad, empleando ocho lámparas de tubos fluorescentes de $30 \mathrm{~W}$ cada una, con características similares a la luz de día. Las plantas que fueron utilizadas, Phragmites australis (carrizos) se extrajeron de una misma planta madre y con un sólo brote del mismo tamaño que fue tomado de un humedal de flujo horizontal ubicado en los Viveros de Coyoacán de la Ciudad de México. Un brote de 1.5 $\mathrm{cm}$ de altura se plantó a $10 \mathrm{~cm}$ de profundidad en el reactor $R A_{1}$ y otro similar en el $R B_{1}$. Los reactores $R A_{2}$ y $R_{2}$ se emplearon como control sin planta. Para la medición del pOR (potencial de óxido-reducción) se utilizaron electrodos comerciales de $\mathrm{Ag} / \mathrm{AgCl}-\mathrm{Pt}$. Se colocaron a diferentes profundidades $(2,10$ y $30 \mathrm{~cm})$, con respecto de la parte superior de los reactores y se tomaron mediciones con un sistema de adquisición de datos computarizado durante las 24 horas de cada día, siendo almacenados para su análisis posterior. En todos los reactores se midieron los parámetros de fósforo total, DQO, potenciales redox, $\mathrm{pH}$ y temperatura siguiendo las metodologías estandarizadas que se presentan en la literatura (13) y con un potenciómetro comercial ORIÓN, Modelo 720A, de acuerdo con las condiciones del fabricante. La temperatura del cuarto donde se encontraba el sistema fue medida con un termómetro de la marca "Brannan".

Tabla 1. Estratos de material de soporte en las columnas de estudio (11)

\begin{tabular}{ccc}
\hline $\begin{array}{c}\text { Diámetro granular } \\
(\mathbf{m m})\end{array}$ & $\begin{array}{c}\text { Grosor del estrato } \\
(\mathbf{c m})\end{array}$ & $\begin{array}{c}\text { Altura relativa a la } \\
\text { parte inferior de la } \\
\text { columna }(\mathbf{m m})\end{array}$ \\
\hline $4-8$ & 20 & 110 \\
$0.5-4$ & 60 & 90 \\
$4-8$ & 10 & 30 \\
$12-16$ & 20 & 20 \\
\hline
\end{tabular}

\subsection{Características del agua residual}

La alimentación del sistema se llevó a cabo en dos etapas, la primera fase experimental se realizó con un agua residual sintética que tenía una $\mathrm{DQOsoluble} \mathrm{=}$ $450 \mathrm{mg} \mathrm{O} / 2$ con una relación C:N:P de 30:1:0.04, empleando sacarosa como fuente de carbono, $\mathrm{C}_{12} \mathrm{H}_{22} \mathrm{O}_{11}$; sulfato de amonio como fuente de nitrógeno, $\left(\mathrm{NH}_{4}\right)_{2} \mathrm{SO}_{4}$ y fosfato de sodio dodecahidratado como fuente de fósforo, $\mathrm{Na}_{3} \mathrm{PO}_{4} \cdot 12 \mathrm{H}_{2} \mathrm{O}$. Para la segunda fase experimental se redujo la $D Q O$ soluble a $225 \mathrm{mg} \mathrm{O} / \mathrm{L}$ empleando los mismos reactivos. El agua sintética se preparó diariamente disolviendo 18 $\mathrm{g}$ de sacarosa, $1.2 \mathrm{~g}$ de sulfato de amonio y $0.12 \mathrm{~g}$ de fosfato de sodio dodecahidratado en $40 \mathrm{~L}$ de agua de la llave para evitar problemas de descomposición en el tanque de almacenamiento y en las tuberías de alimentación. Para la segunda etapa, sólo se varió la cantidad de sacarosa pesando $9 \mathrm{~g}$ de este elemento, dando una relación $C: N: P$ de 15:1:0.04. 


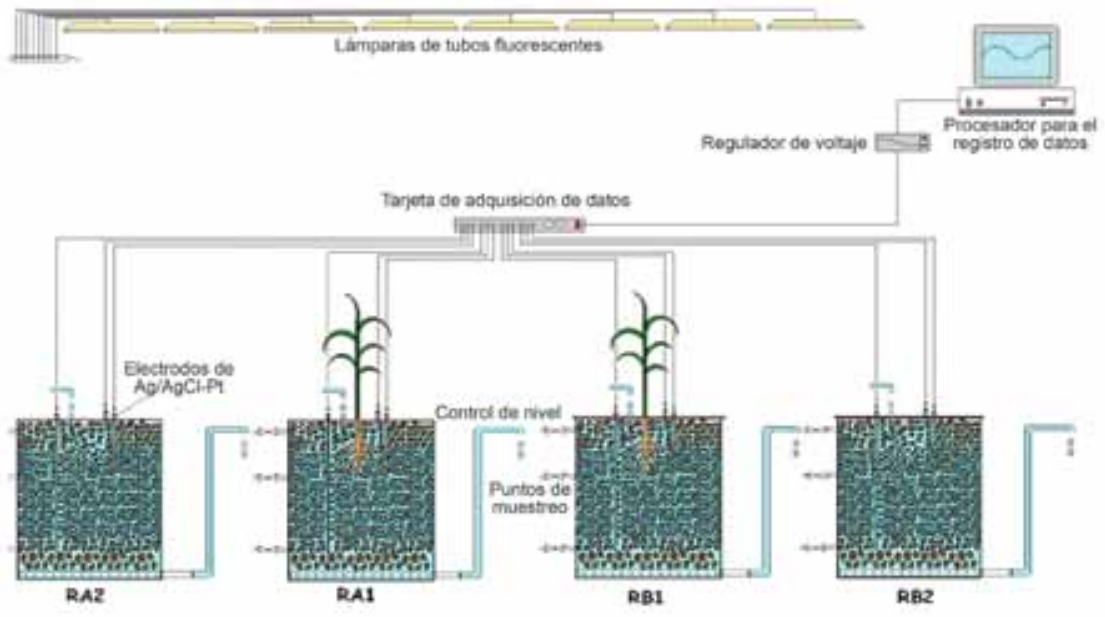

Figura 1. Diagrama del sistema experimental completo (1)

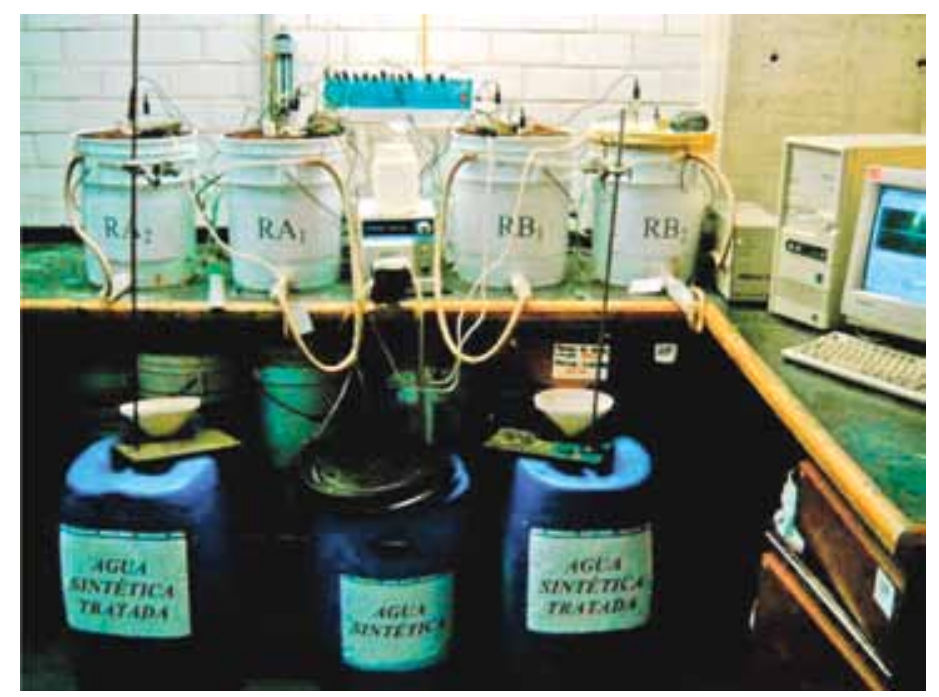

Figura 1a. Sistema experimental

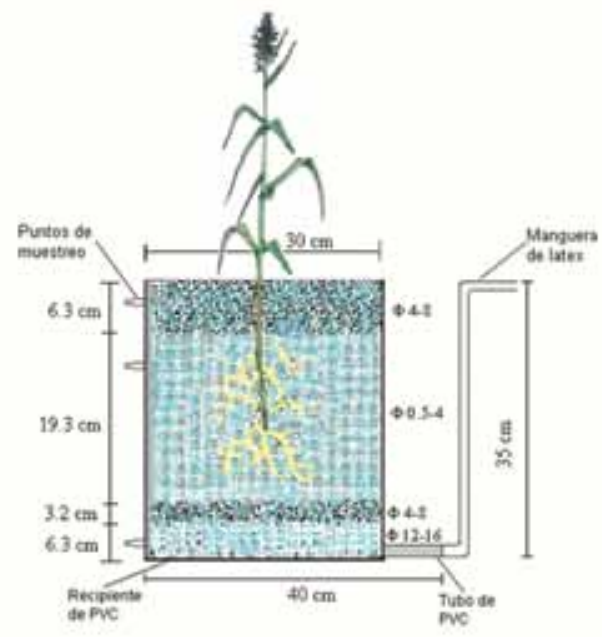

Figura 2. Detalle de la construcción de los reactores (1)

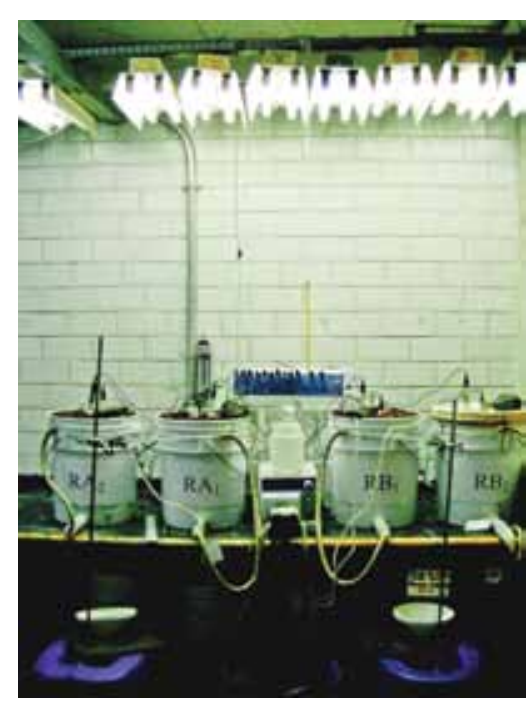

Figura 1b. Sistema experimental con iluminación

\subsection{Diseño experimental}

De acuerdo con lo señalado arriba, el diseño experimental fue el siguiente:

Efecto de las macrofitas en la remoción de fósforo:

\begin{tabular}{lcc}
\hline & $\begin{array}{c}\text { Presencia } \\
\text { de macrofita }\end{array}$ & $\begin{array}{c}\text { Ausencia de } \\
\text { macrofita }\end{array}$ \\
\hline Reactor $\mathrm{RA}_{1}, \mathrm{RB}_{1}$ & $\mathrm{X}$ & \\
Reactor $\mathrm{RA}_{2}, \mathrm{RB}_{2}$ & & $\mathrm{X}$ \\
Concentración alta de carbono & $\mathrm{X}$ & $\mathrm{X}$ \\
Concentración baja de carbono & $\mathrm{X}$ & $\mathrm{X}$ \\
\hline
\end{tabular}

Efecto de las macrofitas en la remoción de carbono medido como DQO:

\begin{tabular}{ccc}
\hline & $\begin{array}{c}\text { Presencia } \\
\text { de macrofita }\end{array}$ & $\begin{array}{c}\text { Ausencia de } \\
\text { macrofita }\end{array}$ \\
\hline Reactor $\mathrm{RA}_{1}, \mathrm{RB}_{1}$ & $\mathrm{X}$ & \\
Reactor $\mathrm{RA}_{2}, \mathrm{RB}_{2}$ & & $\mathrm{X}$ \\
Concentración alta de carbono & $\mathrm{X}$ & $\mathrm{X}$ \\
Concentración baja de carbono & $\mathrm{X}$ & $\mathrm{X}$ \\
\hline
\end{tabular}

\subsection{Metodología para el seguimiento del sistema a escala de laboratorio}

Se evaluaron los parámetros mencionados en el agua residual del influente y del efluente de cada uno de los reactores del sistema a escala de laboratorio, los cuales fueron promedios de mediciones hechas por triplicado. La evaluación estadística del diseño experimental se realizó empleando el paquete Statgraphics versión 5. 


\section{Resultados y Discusión}

La Figura 3 muestra el comportamiento de la disminución de fósforo en las dos etapas (para contenidos iniciales de materia orgánica medidos como demanda química de oxígeno, DQO soluble, en el agua residual sintética de 450 y $225 \mathrm{mg} \mathrm{O} / \mathrm{L}$ ) empleando las eficiencias de remoción. Debido a algunos problemas de operación (fallas eléctricas, estancamientos de agua en los reactores), que se tuvieron en algunos días de la segunda etapa, esos valores no reflejan un comportamiento claro, por lo que no se tomaron en cuenta para el análisis, empleándose solamente los de los primeros días donde los reactores tuvieron una tendencia estable (días de operación 15 al 28).

Los valores de fósforo obtenidos indican que hay una disminución de este elemento en forma de fosfatos en todos los efluentes de los reactores. Es claro que tanto la biopelícula como las propias plantas están haciendo uso de esta fuente como macronutriente esencial para su crecimiento y desarrollo. En el caso de los microorganismos que están presentes en el material de soporte como biopelícula, este nutriente es esencial ya que lo asimilan para así poder llevar a cabo los procesos metabólicos para su desarrollo y su reproducción incorporándolo a sus tejidos y como la tasa de crecimiento de los mismos se lleva a cabo muy rápido los microorganismos juegan un papel muy importante en la remoción de este elemento.

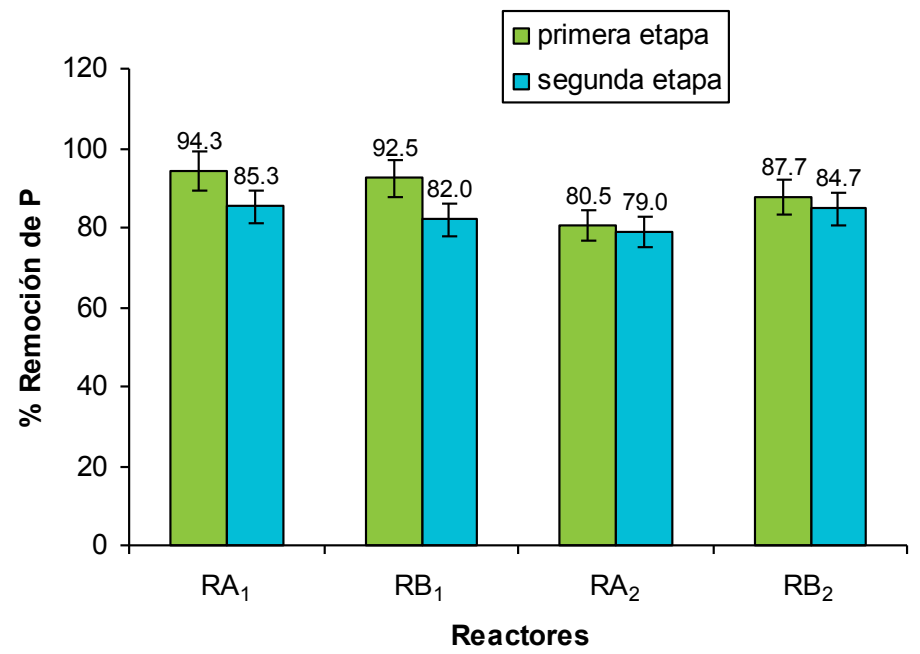

Figura 3. Porcentajes de remoción de fósforo promedio de cada reactor durante las dos etapas en estudio

Como se observa, los reactores con planta $\left(R A_{1}\right.$ y $\left.R B_{1}\right)$ muestran claramente una remoción mayor que los reac- tores que no la tienen $\left(R_{2}\right.$ y $R_{2}$ ) (debido justamente a la presencia de la macrófita), por lo que se puede observar que la planta juega un papel importante en la remoción de fósforo, ya que los cuatro reactores tienen el mismo material de soporte y también biopelículas microbiológicas similares. Sin embargo, dentro de los reactores se llevan a cabo otros mecanismos de remoción de fósforo además de la toma de este nutriente por la biopelícula y las propias plantas. Estos mecanismos son de tipo químico, tales como la formación de complejos con aluminio y con hierro a valores de $\mathrm{pH}$ ligeramente ácidos. El fósforo puede ser adsorbido en los hidróxidos de Fe y Al de la roca volcánica en condiciones ácidas y pueden precipitar como fosfatos de Fe y Al (14). El fósforo forma comúnmente complejos insolubles con el hierro oxidado (15). Se esperaba que en los reactores con planta se viera un diferencia notable en la remoción de fósforo con respecto de los reactores que no la tenían, debido a una mayor disponibilidad de oxígeno disuelto aportado por la raíz de la plantas en la zona de la rizosfera. Esto permitiría una buena oxigenación para los microorganismos aerobios que se encuentran en los alrededores de la raíz, por lo que habría una buena eficiencia en la remoción de fósforo por la planta y por microorganismos. Sin embargo, el aporte de la planta es todavía muy bajo, ya que es una sola y se encuentra en su primera etapa de crecimiento. El comportamiento es muy similar, tanto en los reactores que tienen planta como en los que no la tienen.

También se esperaba que la diferencia de $\mathrm{C} ; \mathrm{N} ; \mathrm{P}$ mostrara una mayor eficiencia en la remoción de fósforo total, particularmente para la etapa de $450 \mathrm{mgO}_{2} / \mathrm{L}$, ya que los microorganismos y la planta deberían tener el suficiente sustrato para su crecimiento y así una mejor asimilación del fósforo. Sin embargo, como una mayor concentración de materia orgánica (DQO) incrementó el consumo de oxígeno necesario para oxidarla y, por ende, se crearon condiciones más reductoras en los reactores, esto afectó la presencia de especies oxidadas de Fe para la formación de complejos insolubles de fósforo.

Estos resultados muestran, en primer término, que en todos los reactores hubo una remoción de fósforo y que esta remoción fue significativamente mayor en los reactores con planta $\left(R A_{1}\right.$ y $R B_{1}, 93 \%$ en promedio para ambos reactores) $(p<0.05,0.01)$. Como se ob- 
serva en la Figura 3, el porcentaje de remoción de fósforo en los reactores que tienen planta es $5 \%$ mayor que en los controles. Esto significa que entre el 80 y el $87 \%$ del fósforo se elimina por mecanismos ajenos a la planta. Estos mecanismos pueden ser bioquímicos, físicos o químicos. La cantidad de fósforo removido por medio de las plantas parece haber sido inferior al que se alcanza por precipitación (7). Esto deberá probarse a futuro con experimentos sin microorganismos ni plantas (sistema estéril).

La Tabla 2 muestra los valores promedio del pOR para el reactor con planta $R_{1}$. Los valores del potencial redox en la zona de la rizosfera la la profundidad de $10 \mathrm{~cm}$ ) son positivos a diferencia de los obtenidos a 2 y a $30 \mathrm{~cm}$, lo que indica que el oxígeno generado en esta zona fue el suficiente para elevar los potenciales. Este fenómeno puede ser exclusivo de este reactor ya que en el reactor sin planta $\left(R_{2}\right)$ se obtuvieron $p O R$ negativos en todos los casos.

Tabla 2. Valores promedio del $p O R$ para las diferentes profundidades (sistema $R A$ )

\begin{tabular}{ccc}
\hline Profundidad & $\begin{array}{c}\text { Reactor RA } \\
\text { (con planta) }\end{array}$ & $\begin{array}{c}\text { Reactor RA } \\
\text { (sin planta) }\end{array}$ \\
\hline $2 \mathrm{~cm}$ & $-151 \mathrm{mV}$ & $-171 \mathrm{mV}$ \\
$10 \mathrm{~cm}$ & $+213 \mathrm{mV}$ & $-191 \mathrm{mV}$ \\
$30 \mathrm{~cm}$ & $-91 \mathrm{mV}$ & $-141 \mathrm{mV}$ \\
\hline
\end{tabular}

La Tabla 3 muestra el porcentaje de la remoción en la DQO, la cual en los reactores con planta fue ligeramente mayor. Los resultados obtenidos muestran cambios similares entre las dos etapas estudiadas (DQO diferente), dado que las plantas necesitan el fósforo como macronutriente para llevar a cabo sus procesos metabólicos independientemente de la fuente de carbono. Debe recordarse que los reactores sin planta tienen microorganismos adheridos al material de empaque formando una biopelícula, que es la responsable de la mayor parte de la degradación de la materia biodegradable, aún cuando los microorganismos remueven material orgánico en los reactores sin planta.

Respecto de la remoción de carbono medido como DQO, hubo diferencias estadísticamente significativas debido a la presencia de la macrofita, así como a la concentración inicial de carbono $(p<0.05$ para la presencia de la planta y $p<0.05,0.01$ para la concen- tración inicial de carbono), siendo ligeramente mayor en aquellos reactores con planta, indicando que ésta sí tiene un efecto depurador adicional a las comunidades microbiológicas que proliferan en los reactores.

Tabla 3. Valores promedio de la remoción de la $D Q O$ promedio de cada reactor durante las dos etapas 450 y $225 \mathrm{mg} / \mathrm{L}$, respectivamente)

\begin{tabular}{|c|c|c|c|c|}
\hline \multirow{2}{*}{$\begin{array}{c}\% \text { de } \\
\text { remoción }\end{array}$} & $\mathrm{RA}_{1}$ & $\mathbf{R B}_{1}$ & $\mathbf{R A}_{2}$ & $\mathbf{R B}_{2}$ \\
\hline & \multicolumn{2}{|c|}{ Con planta } & \multicolumn{2}{|c|}{ Sin planta } \\
\hline Etapa 1 & 62.19 & 64.15 & 51.26 & 57.54 \\
\hline Etapa 2 & 66.24 & 67.84 & 65.11 & 64.81 \\
\hline
\end{tabular}

Aunque el aporte de la planta es mínimo en la remoción de fósforo, no deja de ser importante su aporte en un humedal, como se observa con los pOR y la remoción de $\mathrm{DQO}$.

\section{Conclusiones}

Con base en los resultados obtenidos en esta investigación, para alcanzar el objetivo planteado de evaluar la eliminación de fósforo en reactores biológicos de flujo vertical a escala de laboratorio empacados con escoria volcánica que simulan un humedal artificial, se puede concluir lo siguiente:

Se obtuvo una remoción de fósforo en todos los reactores con y sin planta con una eficiencia promedio de aproximadamente el $90 \%$. Los reactores con planta alcanzaron un $93 \%$ aproximadamente y los reactores sin planta un $86 \%$ aproximadamente, lo que significa que los reactores con una planta joven aportan un $7 \%$ más en la remoción.

Se observó que el efecto en la variación de la concentración de la materia orgánica medida como DQO no influyó en la remoción de fósforo (450 versus 225 $\mathrm{mg} \mathrm{DQO}$ soluble $\mathrm{L}^{-1}$ ), lo que sugiere que los mecanismos principales en la vía de remoción de fósforo podría estarse llevando a cabo principalmente por reacciones de adsorción, formación de complejos y precipitación con aluminio y hierro o con otros metales que estén en el medio.

La presencia de la planta y su aporte de oxígeno en la zona de la rizosfera elevaron los potenciales vol- 
viéndolos positivos, lo cual disminuyó las condiciones reductoras (potenciales redox negativos) que se observaron a los 2 y $30 \mathrm{~cm}$.

\section{Reconocimientos}

Este proyecto fue financiado con fondos del CONACYT, Proyecto UNAM-Universidad Juárez Autónoma de Tabasco, DACB) "Depuración de aguas residuales domésticas usando humedales artificiales", Dirección Adjunta de Desarrollo Regional, Sistema de Investigación Golfo de México, SIGOLFO, Proyecto Clave 00-06-016-V. El segundo autor agradece al Consejo Nacional de Ciencia y Tecnología la beca de maestría otorgada. Los autores agradecen al personal del entonces Programa de Ingeniería Química Ambiental y de Química Ambiental de la UNAM por su valioso apoyo logístico y técnico.

\section{Referencias}

1 Guido-Zárate, A. 2006. Estudio de los potenciales de óxido-reducción en reactores biológicos que simulan un humedal artificial. Tesis de Maestría en Ingeniería. Programa de Maestría y Doctorado en Ingeniería (Ingeniería Ambiental), UNAM. México D.F. México.

2 Durán-Domínguez-de-Bazúa, C., Luna-Pabello, V.M. 1998. Humedales artificiales de flujo horizontal o vertical, procedimiento para tratar aguas residuales. Solicitud de Registro de Patente: Diciembre 15, 1998. Patente Núm. 210924 otorgada el 21 de octubre de 2002. Cesión irrestricta de derechos a la UNAM. Instituto Mexicano de la Propiedad Industrial. Dirección Divisional de Patentes. México, D.F. México.

3 SEMARNAT. 2006. Página de redes internacionales. www.semarnat.gob.mx

4 Luna-Pabello, V.M., Miranda-Ríos, M. 2001. Estado del arte y perspectivas de aplicación de los humedales artificiales de flujo horizontal en México. Serie: Tratamiento biológico de aguas residuales. UNAM. México, D.F. México.

5 Metcalf y Eddy, Inc. 1991. Wastewater Engineering. Treatment, disposal, and reuse. $3^{a}$. Edición. McGraw Hill. Nueva York, EEUUA.

6 Hammer, D. A., Bastian R. K. 1991. Wetlands ecosystems: Natural water purifiers? Constructed wetlands for wastewater treatment. Municipal, industrial and agricultural. Tercera edición. Lewis Publishers. Boca Raton, FL, EEUUA.

7 IWA. 2001. Constructed wetlands for pollution control. Processes, performance, design and operation. IWA Specialist Group on Use of Macrophytes in Water in Water Pollution Control. Scientific and Technical Report Series. International Water Association, IWA Pub. Londres, Inglaterra.

8 Rodríguez-Cruz, A., Varela-Montellano, E. 2003. Comportamiento dinámico de dos sistemas de tratamiento de aguas residuales de tipo humedal artificial de flujo horizontal y vertical. Tesis profesional (Ingeniería Química). Facultad de Estudios Superiores Zaragoza, UNAM. México D.F. México.

9 Lara-Borrero, J.A. 1999. Depuración de aguas residuales municipales con humedales artificiales. Tesis de Maestría en Ingeniería y Gestión Ambiental. Instituto Catalán de Tecnología, Universidad Politécnica de Cataluña. Barcelona, España.

10 Soto-Esquivel, M.G. 2003. Efecto de la generación de oxígeno fotosintético en un sistema sólido-líquido-gas. Tesis de Maestría en Ingeniería. Programa de Maestría y Doctorado en Ingeniería (Ingeniería Ambiental). UNAM. México, D.F. México.

11 Fenoglio-Limón, F.E. 2000. Bases para la construcción de un reactor biológico experimental basado en los sistemas de humedales artificiales de flujo vertical. Tesis profesional (Ingeniería Química), Facultad de Química, UNAM. México, D.F. México.

12 Fenoglio-Limón, F.E. 2003. Fenómenos de transferencia de oxígeno por convección en sistemas que simulan humedales artificiales utilizando columnas empacadas. Tesis de Maestría en Ciencias. Programa de Maestría y Doctorado en Ciencias Químicas (Orientación: Química Ambiental). UNAM. México, D.F. México.

13 Reyes-Luz, M. I. 2006. Remoción de fósforo en un sistema de humedales artificiales a escala de laboratorio. Tesis profesional (Ingeniería Química). Facultad de Química, UNAM. México D.F. México.

14 Faulkner, S.P., Richardson, C.J. 1991. Physical and chemical characteristics of freshwater wetland soils. Wetlands for wastewater treatment. Municipal, industrial and agricultural. Tercera edición. Lewis Publishers. Boca Raton, FL, EEUU.

15 Kadlec, R.H., Knight, R.L. 1996. Treatment Wetlands. Lewis-CRC Press. Boca Raton, FL, EEUU.

16 Durán-de-Bazúa, C. (responsable), Haberl, R., Kreiner, I., Krishnan-Ranjani, Luna-Pabello, V.M., Ramírez-Burgos, L.I. (Profesores); Bernal-González, M., Esponda-Aguilar, P.L., Fenoglio-Limón, F.E., García-Gómez, R.S., García-Vázquez, L., Guzmán-Aguirre, S., GuzmánLópez, P., Juárez-Méndez, C.H., Kneidinger, Ch., Millán-Hernández, S.E., Miranda-Ríos, M., Ramírez-Carrillo, H.F., Mejía-Chávez, A.G., Padrón-López, R.M., Ramírez-Burgos, L.I., Rodríguez-Cruz, A., Rodríguez-Monroy, J., Salinas-Castillo, N.V., Schaller, P., Soto-Esquivel, M.G., Varela-Montellano, E. (Estudiantes). 1999. Humedales artificiales en México: Desarrollo, situación actual y aplicaciones potenciales. VOL. 6, SERIE: QUÍMICA AMBIENTAL DEL AGUA. Pub. Prog. Ing. Quim. Amb. y Quim. Amb. ISBN 968-36-9443-8. Facultad de Química, UNAM. 185 pags. 200 ejemplares, 1a. Ed. (1999), 194 pags. 500 ejemplares, 2a. Ed. (2001), 205 pags. 500 ejemplares, $3 a$. Ed. (2012). En prensa (Versión corregida y aumentada). México, D.F. México. 